\title{
Assessment of Pseudocyst in Pancreas among Patients attending General Hospital in Kakinada: A Prospective Study
}

\author{
Damacharla Basavaiahchowdary ${ }^{1}$, P. Premkamal ${ }^{2}$

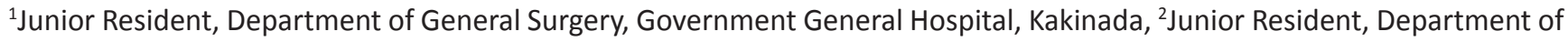 \\ General Surgery, Government General Hospital, Kakinada, Andhra Pradesh, India
}

Corresponding author: Dr R. Mahalakshmi M.S, Professor of Surgery, Department of General Surgery, Kakinada, Andhra Pradesh, India

DOI: http://dx.doi.org/10.21276/ijcmsr.2019.4.2.13

How to cite this article: Damacharla Basavaiahchowdary, P. Premkamal. Assessment of pseudocyst in pancreas among patients attending general hospital in Kakinada: a prospective study. International Journal of Contemporary Medicine Surgery and Radiology. 2019;4(2):B56-B60.

\section{A B S T R A C T}

Introduction: Pancreatic pseudocyst is a circumscribed collection of fluid rich in pancreatic enzymes, blood and necrotic tissue typically located in the lesser sac of the abdomen. It is the result from the complication of pancreatitis. With the advancements in surgical techniques, newer techniques such as internal drainage via cystogastrotomy and cystjejunostomy have been well established and the permanent resolution of these cysts has been reported in $91 \%$ to $97 \%$ of the cases. Hence, the aim of the present study was to assess the etiology, different modalities in management and outome of pseudocyst of pancreas upto from 2 months to 2 years in a Government hospital, Kakinada.

Material and Methods: All the patients presented with recurrent attacks of abdominal pain and epigastric lump. All 50 cases admitted to the Government General Hospital, Kakinada was observed from June 2016 to October 2018. The examination was based on the history and detailed analysis of abdominal pain and the epigastric lump. Ultrasound and CECT abdomen was also performed in all these patients. Blood analysis including urea, sugar grouping and typing, serum amylase and lipase liver function tests was also performed.

Results: Among 50 cases, 40 patients are presented with chronic pancreatitis, and ten are presented with acute pancreatitis. Of the 50 patients 40 members presented with upper abdominal pain which was mainly severe aching in nature with on and off attacks. 25 patients were presented with abdominal lump.

Conclusion: It is more prevalent among males. The most common cause of pseudocysts was found to be chronic pancreatitis. The most common etiology is alcohol and the most common feature is abdominal pain.

Keywords: Pseudocysts, Pancreas, Abdominal Pain, Pancreatitis

\section{INTRODUCTION}

The first description of pseudopancreatic cyst was done in $1761 \mathrm{AD}$ by Cannon et al. At the beginning of $20^{\text {th }}$ century, Eugene Opie was the first to distinguish the true pancreatic cysts, which are by definition, lined by epithelium, from pseudocysts, which are surrounded by a wall composed of collagen and granulation tissue. Atlanta classification was done in 1993 after the two centuries of the first description. The Atlanta classification consists of 4 distinct disease entities. ${ }^{1,2}$

Acute pancreatic pseudocysts, which arise as sequelae of acute pancreatitis or trauma, consists of granulation tissue and extracellular matrix; chronic pancreatic pseudocysts which arises sequelae of chronic pancreatitis and are likewise surrounded by a wall; pancreatic abscess, which are intraabdominal collections of pus immediately adjacent to the pancreas, without any large areas of necrosis. Acute fluid collections, pancreatic pseudocysts, and a pancreatic abscess can be distinguished from one another by the history, imaging studies of the wall of the abnormality and its contents, and if necessary, a needle aspiration of the material. ${ }^{3,4}$

The pancreas develops as two separate primordial buds, the dorsal and the ventral, each as an out-growth of endoderm at the junction of foregut and midgut. The pancreas is a composite gland having exocrine acini which discharge their secretions into the duodenum to assist digestion and groups of endocrine cells, the islets of Langerhans, whose tole is in carbohydrate metabolism. A chronic collection of pancreatic fluid surround by an unepithelialized wall of granulation tissue and fibrosis is referred to as pseudocyst. It is a most common cystic lesion of pancreas accounting for 75\%-80\% of such masses. ${ }^{5,6}$

Pseudocysts occur in $10 \%$ of patients with acute pancreatitis, $20-38 \%$ of patients with chronic pancreatitis, and $4 \%$ of patients with pancreatic trauma. It occurs most commonly in males than in females. In children, it follows after abdominal trauma. Following acute and chronic pancreatitis there is a pancreatic duct disruption which leads to pancreatic duct leak with extravasation of pancreatic juice results in pancreatic fluid collection. ${ }^{7,8}$

Throughout 3-4 weeks, the pancreatic fluid collection 
is sealed by an inflammatory reaction that leads to the development of the wall of acute granulation tissue without much fibrosis leading to acute pseudocyst. In contrast, chronic pseudocyst has well-defined wall but arise in patients with chronic pancreatitis and lack an antecedent episode of acute pancreatitis. ${ }^{9}$

It is the collection of pancreatic fluid surrounded by fibrosis and un epitheliased wall of granulation tissue. It contributes about $75 \%$ of cystic pancreatic lesions. It is the most common complication of chronic pancreatitis. It can occur anywhere between mediastinum and scrotum. The most common site is a lesser sac i.e. anterior pararenal space. Pseudocysts due to chronic pancreatitis are located in gland substance. Its incidence is 10 to $20 \%$ in acute pancreatitis and about 22 to $40 \%$ in chronic pancreatitis. The most common cause is alcohol. Pancreatic fluid collection is due to duct leak and pancreatic juice extravasation. The most common symptom is abdominal pain abdominal lump, weight loss, jaundice, and fever. Complications are infection, rupture, pain haemorrhage and obstruction. ${ }^{10}$

More than $50 \%$ of acute pancreatic fluid collections resolve while in only $5-10 \%$ patients with acute pancreatitis a pseudocyst is formed. Isolated injury due to blunt abdominal trauma or transaction of the main pancreatic duct during pancreatic resection can result in extravasation of pancreatic enzymes and local inflammation response. Initially, cyst wall is thin, but later it got fibrosis and thickened and matured. Pseudocysts fluid has a high concentration of amylase, lipase, and enterokinase such as trypsin. These concentrations reflect the origin of a lesion from extravasated pancreatic exocrine secretions resulting from pancreatic ductal disruption. ${ }^{11}$ Hence, the aim of this study was to assess the etiology, different modalities in management and outome of pseudocyst of pancreas upto from 2 months to 2 years.

\section{MATERIAL AND METHODS}

The present study was a cross-sectional study which was performed on 50 patients of pseudopancreatic cysts for 24 months i.e. from October 2016 to October 2018. All the patients presented with recurrent attacks of abdominal pain and epigastric lump. All 50 cases admitted to the Government General Hospital, Kakinada was observed from June 2016 to October 2018. The examination was based on the history of the patient which included age and mode of onset of the disease and detailed analysis of abdominal pain and the epigastric lump.

The physical examination was done regarding the nutritional status of these patients, abdominal tenderness and lump and regarding complications of pseudocyst of the pancreas. Ultrasound and CECT abdomen was also performed in all these patients. Blood analysis including urea, sugar grouping and typing, serum amylase and lipase liver function tests was also performed. Different surgeries in the form of cystojejunostomy, cystoduodenostomy, cystogastrostomy, and distal pancreatectomy were done and results analyzed. Normal serum amylase was taken as 23-85 U / $\mathrm{L}$ and normal serum lipase was taken as $0-160 \mathrm{U} / \mathrm{L}$ in the present study.

\section{Cystic fluid amylase}

High concentrations are found in pseudocyst of the pancreas where as low amylase in cystic fluid could suggest either mucinous or serous cystadenoma. These are found to be as follows:

CEA: low in pseudocyst and elevated in tumors.

CA 125: low in pseudocyst and elevated in tumors.

Fluid viscosity is low in pseudocyst and elevated in tumors.

Amylase levels are usually high in pseudocyst and low tumors. Cytology is occasionally helpful in diagnosing tumors, but a negative result doesn't exclude tumors.

CEA > $400 \mathrm{mg} / \mathrm{dl}$ with cystic fluid strongly suggests malignancy.

\section{Liver function test}

About in one-third of patients mild elevation of serum concentration of bilirubin, SGOT and SGPT was also seen.

\section{Surgical procedures}

The primary indication of surgery was intractable pain. It was done under general anesthesia or epidural anesthesia. The abdomen was opened in upper midline incision Findings at operation: in all cases pancreas appear to be firm and large pseudocyst present at, body, head, and tail of the pancreas. Longitudinal anterior gastrostomy is performed between stay sutures and aspiration through the posterior wall of the stomach with a syringe and localization of pseudocyst done. Deep stay sutures are applied along the posterior stomach wall and incision made through the wall, absorbing sutures are used to encompass all layers of the stomach and the pseudocyst wall, anterior gastrostomy closed.

In the case of cystojejunostomy, abdomen opened in layers, the cyst is opened under stay sutures. Cyst fluid drained out, necrotic material removed. Roux en y created, distal loop of jejunum is brought to the cyst, and then side to side anastomosis was done. Hemostasis secured and drain fixed and abdomen was closed in layers.

\section{Imaging studies}

The prime diagnostic tool used in this series was an ultrasound of the abdomen which was done in 50 cases which showed sizeable cystic lesion in the lesser sac. Patients also undergone $\mathrm{CT}$ of the abdomen mostly showing large pseudocyst in the head and body of the pancreas in 14 cases and pseudocyst found at the tail of the pancreas.

\section{RESULTS}

Table no. 1 shows the distribution of data based on age group among the study subjects and it was found that the majority of the patients were from the age group 31-40 years followed by 21-30 years. The least common age group seen was 51-60 years followed by 1-20 years. In table no. 2 it was found that majority of the patients were males $98 \%$ and only $2 \%$ were

\begin{tabular}{|l|c|c|}
\hline Age group & No. of cases & Percentage \\
\hline $1-20$ & 2 & $4 \%$ \\
\hline $21-30$ & 18 & $36 \%$ \\
\hline $31-40$ & 26 & $52 \%$ \\
\hline $41-50$ & 4 & $8 \%$ \\
\hline $51-60$ & 0 & $0 \%$ \\
\hline \multicolumn{2}{|c|}{ Table-1: Shows the distribution of cases based on age group } \\
among the study subjects \\
\hline
\end{tabular}




\begin{tabular}{|l|c|c|}
\hline Gender & No. of cases & Percentage \\
\hline Males & 49 & $98 \%$ \\
\hline Females & 1 & $2 \%$ \\
\hline \multicolumn{2}{|c|}{ Table-2: Shows the distribution of cases based on the gender } \\
among the study subjects \\
\hline
\end{tabular}

\begin{tabular}{|l|l|c|c|}
\hline S.No & Etiology & No. of cases & Percentage \\
\hline 1 & Alcohol & 38 & $76 \%$ \\
\hline 2 & Biliary tract Diseases & 08 & $16 \%$ \\
\hline 3 & Traumatic & 1 & $2 \%$ \\
\hline 4 & Hyperlipidemia & 2 & $4 \%$ \\
\hline 5 & Idiopathic & 1 & $2 \%$ \\
\hline
\end{tabular}

Table-3: Shows the distribution of data based on the etiology among the study subjects

\begin{tabular}{|l|l|c|c|}
\hline S.No & Presentation & No. of cases & Percentage \\
\hline 1 & Abdominal pain & 40 & $80 \%$ \\
\hline 2 & Abdominal lump & 25 & $50 \%$ \\
\hline 3 & Weight loss & 20 & $40 \%$ \\
\hline 4 & Vomiting & 20 & $40 \%$ \\
\hline 5 & Jaundice & 5 & $10 \%$ \\
\hline \multicolumn{3}{|c|}{ Table-4: Shows the distribution of data based on presentation } \\
among the study subjects \\
\hline
\end{tabular}

\begin{tabular}{|l|l|c|c|}
\hline S. No & Type of management & No. of cases & Percentage \\
\hline 1 & Conservative & 16 & $32 \%$ \\
\hline 2 & Cystogastrostomy & 21 & $42 \%$ \\
\hline 3 & Cysto jejunostomy & 8 & $16 \%$ \\
\hline 4 & Lap cystogastrostomy & 1 & $2 \%$ \\
\hline 5 & External drainage & 2 & $4 \%$ \\
\hline 6 & Percutaneous drainage & 2 & $4 \%$ \\
\hline \multicolumn{4}{|c|}{ Table-5: Shows the distribution of data based on the type of } \\
management among the study subjects \\
\hline
\end{tabular}

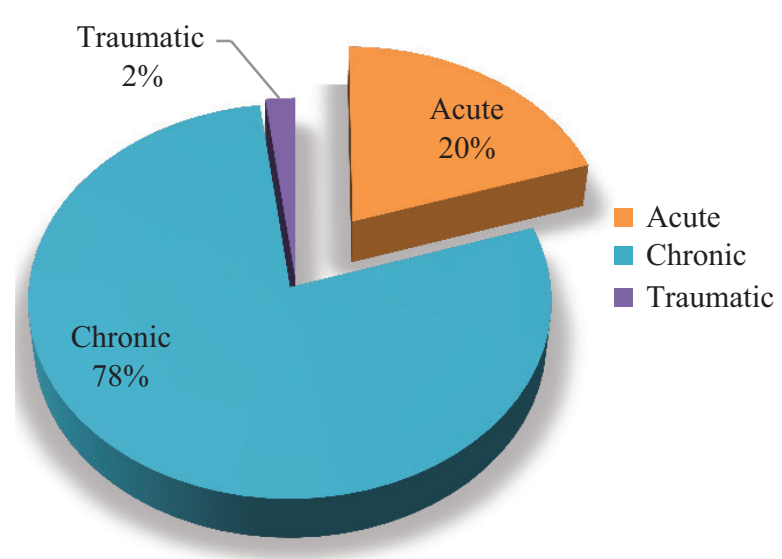

Graph-1: Shows the distribution of data among the study subjects based on various cases of pseudocyst of pancreas

females.

Table no. 3 shows the most common etiology found among study subjects which was found to be alcohol (76\%) followed by biliary tract disease (16\%), hyperlipidemia (4\%), and the least reason was found to idiopathic (2\%) and traumatic (2\%). The most common presentation found among study subjects was abdominal pain (80\%) and abdominal lump

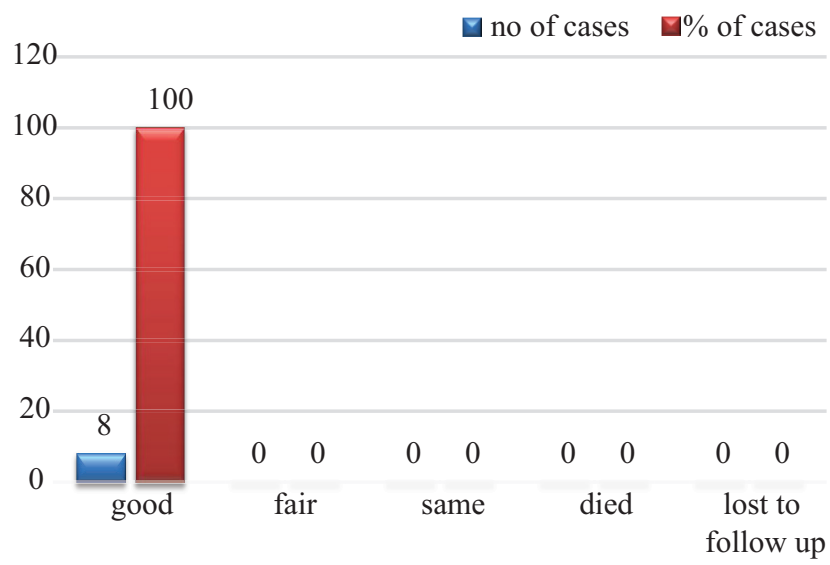

Graph-2: Shows the distribution of data based on the results of cysto jejunostomy among the study subjects

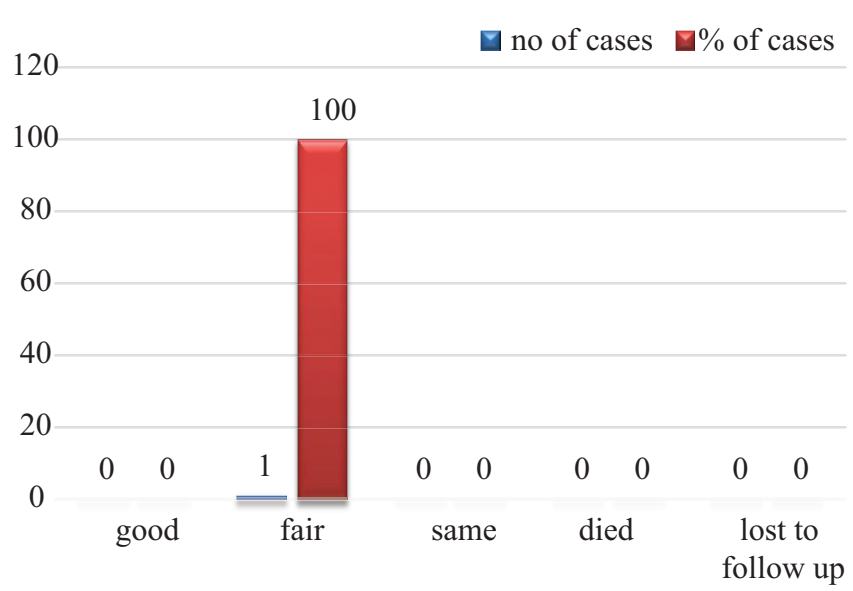

Graph-3: Shows the distribution of data based on the results of laparoscopic cysto gastrostomy among the study subjects

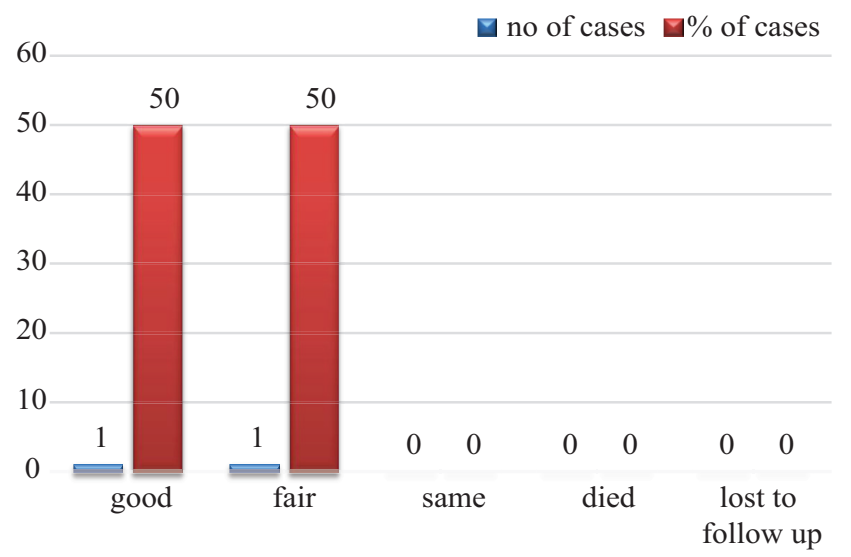

Graph-4: Shows the distribution of data based on the results of external drainage among the study subjects

(50\%) followed by weight loss (40\%) and vomiting (40\%). The least common reason was found to be Jaundice (Table no. 4). Table no. 5 shows that majority of the patients were managed by cystogastrostomy (42\%) and conservatively (32\%). The least number of patients were managed by cysto jejunostomy (16\%) and external drainage (4\%).

In Graph no. 1 the pie chart shows the distribution of data based on various cases of pseudocyst of pancreas and it was found that chronic cases were $78 \%$ followed by acute cases (20\%) and very least were found to be traumatic (2\%). Graph 
no. 2 shows that the majority of the results obtained by doing cysto jejunostomy were found to be good among the study subjects.

Graph no. 3 shows the distribution of data according to the results obtained from laparoscopic cysto gastrotomy. Majority of the results obtained from the subjects were good to fair. It was found from Graph no. 4 that majority of the subjects responded fair after doing external drainage.

Discussion

Pseudocyst of pancreas constitutes one of the major causes of abdominal pain for which patients got admitted in Government General Hospital, Kakinada. Most of the patients admitted in this hospital are from Godavari districts. In this study, cases recorded for a period of 24 months, i.e. from October 2016 to October 2018. These cases were analyzed according to the age of onset, aetiological factors, mode of presentation, management by different surgeries and their outcomes.

In the present study, the incidence of acute pancreatitis was found to be $20 \%$, and for chronic pancreatitis was $78 \%$, and incidence of patients requiring operations was 68\%. In a study done by K. Khanna et al it was found that the incidence of acute pancreatitis was found to be $6-18.5 \%$ which is in concordance with the findings of the present study whereas the incidence of chronic pancreatitis was found to be $20-40 \%$ which is not similar to the present study findings. Another study conducted by Alexander A. Aghdassi et al. it was found that the incidence of acute pancreatitis was found to be $5-16 \%$ followed by incidence of chronic pancreatitis of $20-40 \% .^{12,13}$

In this study, most of the cases were males $98 \%$ patients and only $2 \%$ were females. A study done by Wen.Yao.Yin and $\mathrm{O}$. Malley et al found that males and females were $63.6 \%$ and $69.6 \%$ respectively. Abdominal pain is a consistent finding and is usually located in the epigastrium and upper abdomen quadrants often associated with radiation to back. Patients frequently presented with abdomen mass and sometimes with nausea, vomiting, anorexia, jaundice, and weight loss. ${ }^{14,15}$ In this study, pain in abdomen was the most common complaint present in 40 patients (80\%). A study done by Lee et al found that pain is the most common type of presentation which was seen in $100 \%$ of cases and these findings were relevant to the findings of the present study whereas in a study done by O. Malley et al it was found that pain was the most common presentation which was found in $70 \%$ of cases. A study was done by Sedlack. R et.al revealed that the abdominal lump was the next presentation which was seen in 25 cases (50\%). Other symptoms like vomiting, anorexia, weight loss present in significant number of cases. Fever, ascites and jaundice was present in some patients. ${ }^{16,15,17}$ In the present study, alcoholism accounts for $76 \%$ of cases which is same when compared to other studies. Various countries revealed that alcoholism as the most frequent cause of pancreatitis leading to pseudocyst formation (65$80 \%$ ) followed by biliary tract disease (6- 20\%), trauma (3$10 \%)$ and idiopathic (6-20\%). About 38 cases in this series had a history of alcoholism (76\%). In a study done by D Egidio et.al it was found that alcoholism accounts for $70 \%$ of cases. In another study done by $\mathrm{O}$ Malley et.al it was seen that alcoholism accounts for $78 \%$ of the cases among study subjects and these findings are in concordance with the results of the present study. ${ }^{18}$

Among 50 cases of pseudocyst of the pancreas in the current study, 16 cases were managed conservatively (32\%) which were similar to the findings of the study done by Sanfey $\mathrm{H}$ et al and Trevor Sandy et al where $32 \%$ and $22 \%$ of cases were managed conservatively. Open Cystogastrostomy was done as a prime modality of treatment in about 21 cases (42\%) in which all cases good pain relief after surgery. ${ }^{19,20}$

In this study, Laparoscopic cystogastrostomy was done in 1 case $(2 \%)$ followed by external drainage in 2 (4\%) cases. Also, the percutaneous drainage was done in 2 cases (4\%). In our study, infection of pseudocyst was seen in $4 \%$ cases. Rupture of pseudocyst was not seen in the present study. Several studies have indicated that the size of the cyst and the length of time the cyst has been present are poor predictors of potential for pseudocyst resolution or complications. But in general larger cysts are more likely to become symptomatic or cause complications. However, some patients with larger collections do well; therefore the size of the pseudocyst alone is not an indication for drainage. Postoperatively, the most striking observation was the complete and immediate relief of pain except in 3 cases which has fair relief of pain, which was managed with antibiotics and painkillers.

\section{CONCLUSION}

Pseudocyst of the pancreas is a common complication of chronic pancreatitis which is potentially troublesome clinical entity, whose management depends upon the severity of pancreatic trauma, size and symptoms.

\section{REFERENCES}

1. Imrie CW. Epidemiology, clinical presentation, and behavior of acute pseudocysts. In, Bradley EL. Acute pancreatitis diagnosis and Therapy, New York, Raven Press. 1994:175-9.

2. Kloppel G. Pseudocysts and other non-neoplastic cysts of the pancreas. Semin Diagn Pathol 2000; 17(3):7-15.

3. Alka Bhambri, Nisha Pandey. Pancreatitis in Children: Experience at a Tertiary Care Centre. Interenational Journal of Contemporary Medical Research 2016; 3(1):257-259.

4. Sherlock S, Dooley J. Diseases of the Liver and Biliary system. 11th Ed. Oxford: Blackwell Scientific Publications; 2002:511-516.

5. Dadoukis J, Gamvros O, Aletras H. Intrabiliary rupture of the hydatid cyst of the liver. World J Surg. 1984; 8 (4):786-790.

6. Heider R, Meyer AA, Galanko Ja, Behrns KE. Percutaneous drainage of pancreatic pseudocysts is associated with a higher failure rate than surgical treatment in unselected patients. Ann Surg 1999; 229 (6):787-789.

7. Ridder GJ, Maschek H, Klempnauer J. Favourable prognosis of cystadeno- over adenocarcinoma of the pancreas after curative resection. Eur J Surg Oncol 1996; 22 (1):232-236.

8. Alper A, Ariogul O, Emre A, Uras A, Okten A. Choledochoduodenostomy for intrabiliary rupture of 
hydatid cysts of liver. Br J Surg.1987; 74 (3):243-5.

9. Seewald S, Ang TL, Teng KC, Soehendra N. EUSguided drainage of pancreatic pseudocysts, abscesses and infected necrosis. Dig Endosc. 2009; 21Suppl LS61-65.

10. Zeytunlu M, Coker A, Yüzer Y, Ersöz G, Aydin A, Teke ${ }^{\circ}$ in $\mathrm{O}$, et al. Hydatid acute pancreatitis. Turk J Gastroenterol. 2004; 15 (5):229-32.

11. Cannon JW, Gallery MP, Vollmer CM. Diagnosis and management of pancreas pseudocysts: what is the evidence? J Am Coll Surg. 2009; 209 (2):385-93.

12. Khanna AK. Sathyendra K, Tivvary. Kumar P.Pancreatic pseudocyst: Therapeutic dilemma. Int J Inflam. 2012; 2012:1-7.

13. Aghdassi A, Mayerle J, Kraft M, et al. Diagnosis and treatment of pancreatic pseudocysts in chronic pancreatitis. Pancreas. 2008; 36 (3):105.

14. Yin W, Chen H, Huang S, Lin C, Lin S, Shyu D, Wei C, Lee M, Chou A, Tseng K. Chang Y. The role of surgery in pancreatic pseudocyst. Tzu Chi Med J 2004; 16 (4):359-369.

15. O. Malley VP cannon JP, Postier RG, Pancreatic Pseudocysts, Cause, Therapy, And Results. Am.j.Surg.1985;150 (6):680-682.

16. Lee JK, Stanley RJ, Melson GL, et al. Pancreatic imaging by ultrasound and computed tomography: a general review. Radiol Clin North Am 1979; 17 (1):105.

17. Sedlack R, Affi A, Vazquez-Sequeiros E, Norton ID, Clain JE,Wiersema MJ. Utility of EUS in the evaluation of cystic pancreatic lesions. Gastrointest Endosc 2002; 56 (5): 543-547.

18. D'Egidio A, Schein M. Pancreatic pseudocysts: a proposed classification and its management implications. Br J Surg 1991; 78 (3): 981-984.

19. Sanfey H Agular M Jones Rs. Pseudocyst of Pancreas. A Review of 97 Cases. Amj.Surg.1994; 60 (1):661-668.

20. Sandy JT, Taylor RH, Christensen RM, Scudamore C, Leckie P. Pancreatic pseudocyst. Changing concepts in management. Am J Surg 1981; 141 (3): 574-576.

Source of Support: Nil; Conflict of Interest: None

Submitted: 10-04-2019; Accepted: 01-05-2019; Published online: 22-05-2019 